\title{
Agenesis of the dorsal mesentery presenting in an adolescent
}

\section{A Chacko, F Ismail, S Ellemdin}

Department of Radiology, Steve Biko Academic Hospital and University of Pretoria

A Chacko, MB BCh

F Ismail, FCRad (D) SA, MMed Rad (D)

Department of Internal Medicine, Steve Biko Academic Hospital and University of Pretoria

S Ellemdin, MB BCh, MMed (Int Med)

Corresponding author: F Ismail (drfismail@yahoo.com)

Agenesis of the dorsal mesentery is a rare occurrence that usually presents in children. It is associated with proximal small-bowel malrotation as well as high jejunal atresia with discontinuity of the small bowel. We present a case report of an adolescent presenting with clinical features of proximal small-bowel obstruction (confirmed on imaging) as well as acute pancreatitis. At laparotomy, he was found to have no dorsal mesentery, without small-bowel atresia, and the duodenum was fixed to the posterior abdominal wall. The patient recovered well and remained symptom-free.

S Afr J Rad 2013;17(1):16-18. DOI:10.7196/SAJR.734

\section{Clinical presentation}

A 16-year-old boy presented with acute pancreatitis. His serum amylase level was raised at $292 \mathrm{U} / \mathrm{l}$ (normal 25 - $125 \mathrm{U} / \mathrm{l}$ ) and serum lipase level was raised at $142 \mathrm{U} / 1$ (normal 0 - $60 \mathrm{U} / \mathrm{l}$ ). He also presented with severe vomiting causing dehydration and clinical features in keeping with obstruction of the proximal gastro-intestinal tract. At this stage he was thought to have a congenital abnormality such as a pancreas divisum or an auto-immune pancreatitis. Antibody tests were negative. There was no previous medical or surgical history or any previous presentations to healthcare facilities with similar episodes.

Erect abdominal radiographs (Fig. 1) confirmed proximal bowel obstruction with the presence of dilated small-bowel loops and multiple dynamic air-fluid levels. A contrast meal and follow-through was performed which showed an abnormal configuration of the duodenum at the D2 - D3 junction suggestive of a malrotation with volvulus. The visible proximal duodenum was dilated and the mucosal pattern demonstrated a 'stacked coin' appearance that might have been due to ischaemia (Fig. 2). On delayed images, many small-bowel loops were clustered in the right hypochondrium (Fig. 3).

Computed tomography (CT) with intravenous contrast (Fig. 4) demonstrated the classic sign of whirling of the mesenteric vessels around the superior mesenteric artery (SMA). The normal relationship of the superior mesenteric vein (SMV) on the right of the SMA was also distorted. The proximal duodenal bowel loops were distended and demonstrated diffuse wall enhancement. The pancreatic duct was also found to be prominent (not shown) and this was thought to be secondary to obstruction from the midgut volvulus.

At laparotomy, it was found that the dorsal mesentery was absent, which caused spontaneous intermittent volvulus of the duodenum with intermittent occlusion of the common bile duct, causing pancreatitis. The duodenum was surgically fixed to the posterior abdominal wall and the patient remained asymptomatic thereafter. He has subsequently done well and has had no further recurrence or further symptoms or complications.

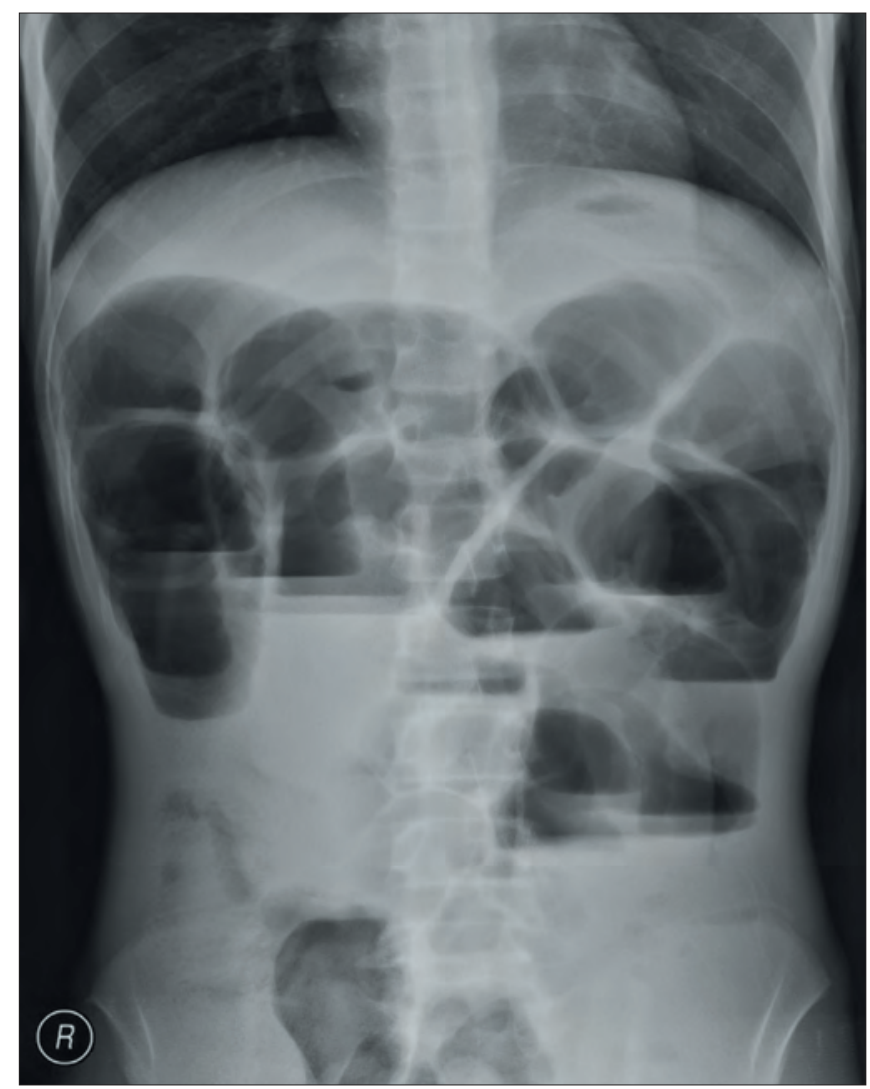

Fig. 1. Erect abdominal radiograph demonstrates dilated proximal small-bowel loops with multiple dynamic air-fluid levels in keeping with proximal smallbowel obstruction. 


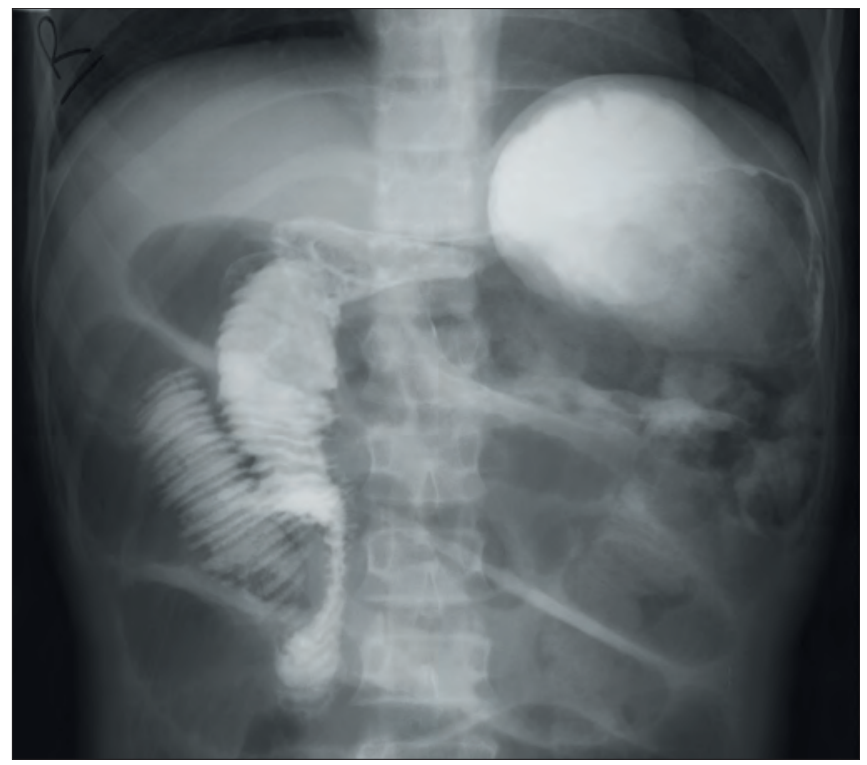

Fig. 2. Iodine contrast meal and follow-through demonstrates malrotation and volvulus of the proximal small bowel with dilation of the proximal duodenum with a 'stacked coin' mucosal pattern, possibly owing to ischaemia.

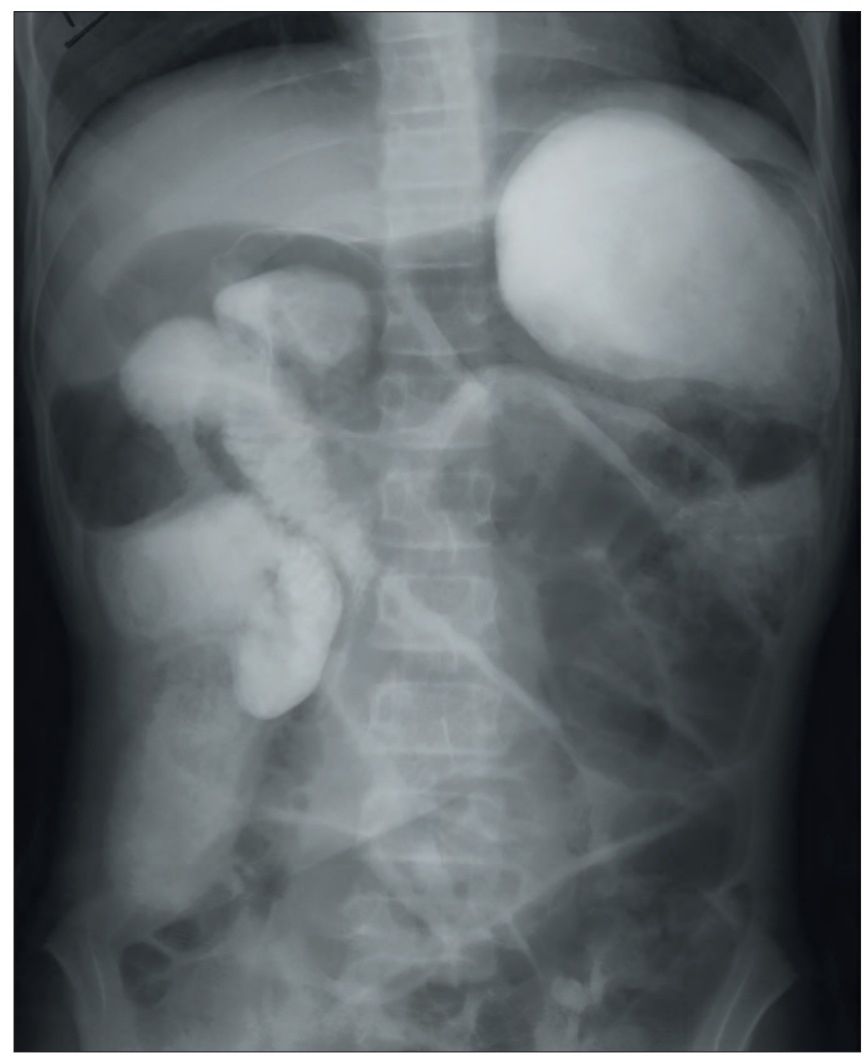

Fig. 3. Iodine contrast meal and follow-through (delayed images). A collection of small-bowel loops is noted in the right hypochondrium.

\section{Discussion}

The development of the mesentery starts when the foregut, midgut and hindgut are in broad contact with mesenchyme of the posterior abdominal wall in the fetus. In the embryo, the connecting tissue bridge then becomes narrow, and the caudal part of the foregut, midgut and most of the hindgut are then suspended from the posterior abdominal wall by the dorsal mesentery. ${ }^{[1]}$

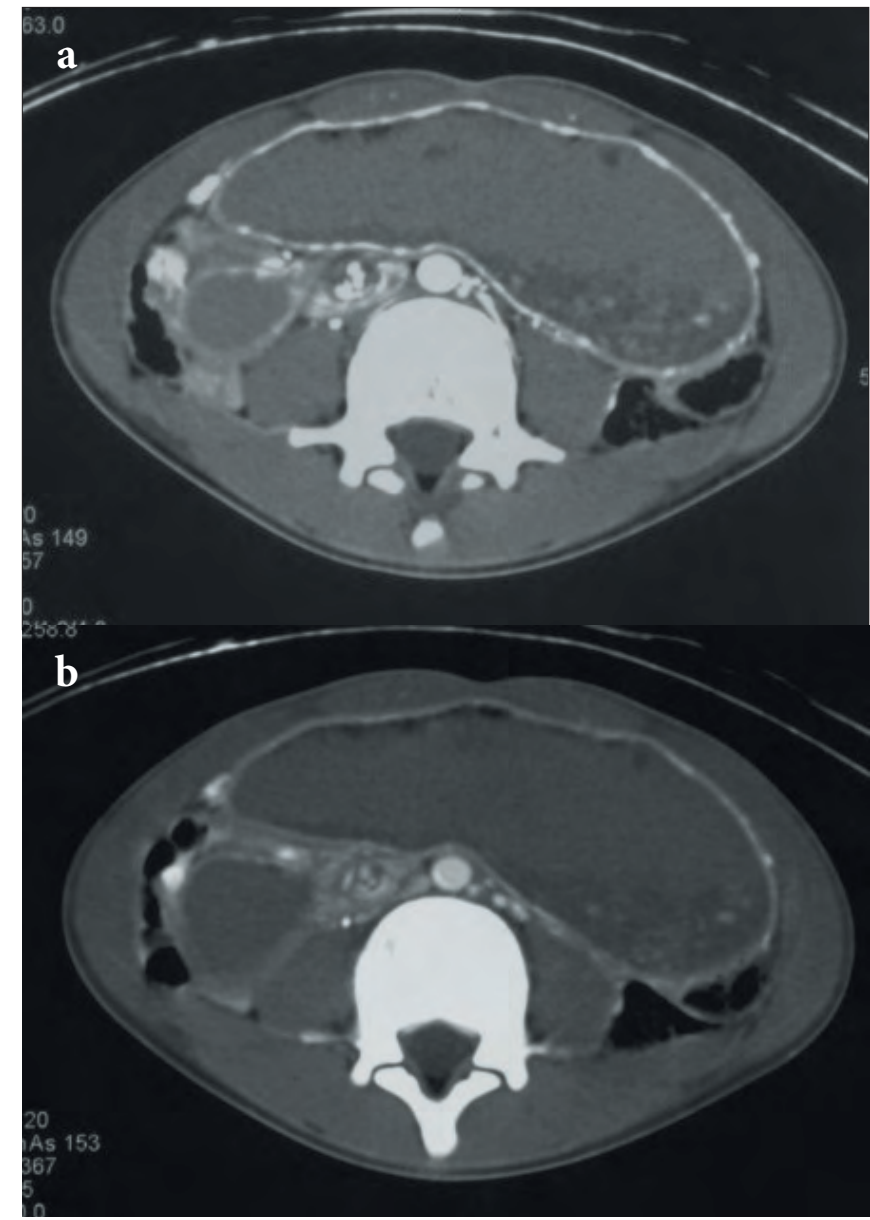

Fig. 4. Computed tomography (CT) with intravenous contrast in (a) the arterial phase and (b) the portal venous phase demonstrates the classic sign of whirling of the mesenteric vessels around the superior mesenteric artery (SMA), in keeping with volvulus.

A ventral mesentery only exists in the terminal part of the oesophagus, stomach and the proximal part of the duodenum. This ventral mesentery gives rise to the lesser omentum, falciform ligament and the visceral peritoneum of the liver. ${ }^{[1]}$

The dorsal mesentery extends from the distal end of the oesophagus to the cloaca distally. It is known as the dorsal mesogastrium or greater omentum in the region of the stomach, the dorsal mesoduodenum in the region of the duodenum, and the dorsal mesocolon in the region of the colon. It is known as the mesentery proper in the region of the jejunal and ileal loops of the small bowel. ${ }^{[1]}$

Mesenteric defects/absences can lead to volvulus of the associated parts of the bowel. These defects can vary from lesions involving a well-defined short segment of the small bowel (known as segmental defects) to lesions involving the entire base of the mesentery (basilar defects). ${ }^{[2]}$

Usually, agenesis of the dorsal mesentery is associated with a high jejunal atresia owing to the vascular ischaemic aetiology of such agenesis. ${ }^{[3-6]}$ This condition then also can present as the entity known as 'apple peel small bowel' which consists of a high jejunal atresia with a discontinuity of the small bowel and a wide gap in the mesentery. ${ }^{[2,3]}$ The distal segment of the ileum is shortened and assumes a helical configuration around a retrograde perfusing vessel, which compensates for the partially absent superior mesenteric artery. ${ }^{[3]}$ An intra-uterine 


\section{CASE REPORT}

vascular accident in late gestation has been accepted as the cause of apple peel atresia and presents with a spectrum of occlusions of one or more branches of the superior mesenteric artery. ${ }^{[3-6]}$

There was a report of isolated agenesis of the dorsal mesentery in 1960 by Blandy ${ }^{[6]}$ and subsequently by Hull et al. ${ }^{[7]}$ in 1975 . However, in the second report, this was associated with the apple peel deformity without small-bowel atresia. A case of absent dorsal mesentery without small-bowel atresia and without apple peel deformity was described by Salvatore et al. in 2006, ${ }^{[8]}$ in which there was complete absence of the dorsal mesentery of the small bowel.

Intermittent volvulus of the duodenum without malrotation is rare and is not easily recognisable. Delays in diagnosis can lead to complications and high morbidity.

Our case is believed to be unique owing to the relatively late presentation of the patient (adolescence versus early childhood) and also highlights the possibility of midgut volvulus occurring outside the neonatal period. Aetiology of the absence of the dorsal mesentery of the duodenum is not clear; an ischaemic cause is unlikely, owing to the absence of atresia.

\section{Conclusion}

Our case report describes a rare entity of absence of the dorsal mesentery in an adolescent, complicated by proximal small-bowel malrotation, volvulus and the absence of small-bowel atresia.

1. Sadler TW. Langman's Medical Embryology, 11th ed. Philadelphia: Lippincott Williams \& Wilkinson, 2010:155, 209-213

2. Black PR, Muller D, Crow J, et al. Mesenteric defects as a cause of intestinal volvulus without malrotation and as the possible primary etiology of intestinal atresia. J Pediatr Surg 1994;29:1339-1343.

3. Zwiren GT, Andrews HG, Ahmann P. Jejunal atresia with agenesis of the dorsal mesentery (apple peel small bowel). J Pediatr Surg 1972;7:414-419.

4. Shorter NA, Georges A, Perenyi A, Garrow E. A proposed classification system for familial intestinal atresia and its relevance to the understanding of the etiology of jejunoileal atresia. J Pediatr Surg 2006;41:1822-1825.

5. Arbella D, Orkina B, Navehb Y, Gurb I, Udassina R. Duodenojejunal atresia with absent dorsa mesentery, choledochal cyst, and malrotation in a premature newborn - a case report. J Pediatr Surg 2006;41:E11-E13.

6. Weber DM, Freeman NV. Duodenojejunal atresia with apple peel configuration of the ileum and absent superior mesenteric artery: Observations on pathogenesis. J Pediatr Surg 1999;34(9):1427-1429.

7. Blandy JP. Neonatal intestinal obstruction from a congenital hole in the mesentery. Br J Surg 1960;48:133

8. Hull JD, Kiesel JL, Warren H, et al. Agenesis of the dorsal mesentery without jejunoileal atresia (apple peel small bowel). J Pediatr Surg 1975;10:277-279.

9. Salvatore C, Alan SLT, Prakash A, Hock LT. Dorsal mesenteric agenesis without small bowel atresia: A rare cause of midgut volvulus in children. J Pediatr Surg 2006 41:e5-e7. 